\title{
Should all patients with hyperparathyroidism be screened for a CDC73 mutation?
}

\author{
Caroline Bachmeier1, Chirag Patel2, Peter Kanowski³ and Kunwarjit Sangla1,4 \\ 1Endocrinology Department, Townsville Hospital, Townsville, Queensland, Australia, ${ }^{2}$ Genetic Health Queensland, \\ Royal Brisbane and Women's Hospital, Herston, Queensland, Australia, ${ }^{3} \mathrm{Clinical}$ Pathology/Histopathology, Sullivan \\ Nicolaides Pathology, Townsville, Queensland, Australia, and 4James Cook University, Townsville, \\ Queensland, Australia
}

Correspondence

should be addressed

to C Bachmeier

Email

carolinebachmeier@

hotmail.de

\section{Summary}

Primary hyperparathyroidism (PH) is a common endocrine abnormality and may occur as part of a genetic syndrome. Inactivating mutations of the tumour suppressor gene $C D C 73$ have been identified as accounting for a large percentage of hyperparathyroidism-jaw tumour syndrome (HPT-JT) cases and to a lesser degree account for familial isolated hyperparathyroidism (FIHP) cases. Reports of CDC73 whole gene deletions are exceedingly rare. We report the case of a 39 year-old woman with PH secondary to a parathyroid adenoma associated with a large chromosomal deletion (2.5 Mb) encompassing the entire $C D C 73$ gene detected years after parathyroidectomy. This case highlights the necessity to screen young patients with hyperparathyroidism for an underlying genetic aetiology. It also demonstrates that molecular testing for this disorder should contain techniques that can detect large deletions.

\section{Learning points:}

- Necessity of genetic screening for young people with hyperparathyroidism.

- Importance of screening for large, including whole gene CDC73 deletions.

- Surveillance for patients with CDC73 gene mutations includes regular calcium and parathyroid hormone levels, dental assessments and imaging for uterine and renal tumours.

\section{Background}

Primary hyperparathyroidism $(\mathrm{PH})$ is a common endocrine pathology with the highest incidence in postmenopausal women, and an overall prevalence of 1-4 per 1000 (1). Despite approximately $90 \%$ of cases arising sporadically, $\mathrm{PH}$ as part of a genetic syndrome including multiple endocrine neoplasiatype 1 or type $2 \mathrm{~A}$, hyperparathyroidism-jawtumour syndrome (HPT-JT), familial hypocalciuric hypercalcaemia (FHH) and familial isolated hyperparathyroidism (FIHP) has been described in the literature (2).

HPT-JT syndrome is an autosomal dominant disorder that predisposes to parathyroid tumours, ossifying lesions of the jaw, and renal and uterine lesions (3). Mutations in the CDC73 gene have been associated with the pathogenesis of HPT-JT syndrome (3). However, mutations in CDC73 have also been identified in parathyroid carcinomas and in families with hyperparathyroidism caused by parathyroid tumours and this clinical variant is classified as FIHP (3).

The CDC73 gene is located at chromosome 1q31.2 and was originally identified in 2002. It has 17 exons encoding the 531 amino acid protein parafibromin (4). Mutations in $C D C 73$ described in the literature include missense, nonsense, and splice site mutations, and small deletions and insertions (3). 
We report the case of a woman with a history of PH secondary to a cystic parathyroid adenoma, associated with a rare genetic mutation. The patient was analysed by SNP chromosome microarray and a deletion of $2.5 \mathrm{Mb}$ at chromosome 1q31.1-1q31.2, which includes the entire CDC73 gene was identified. This deletion was first found in her son, who was being investigated for developmental issues.

\section{Case presentation}

In 2014, a then 36 year-old female was incidentally found to have an elevated corrected calcium level of $2.72 \mathrm{mmol} / \mathrm{L}$ (reference range: $2.10-2.55 \mathrm{mmol} / \mathrm{L}$ ) with an ionised calcium of $1.45 \mathrm{mmol} / \mathrm{L}$ (reference range $1.15-1.30 \mathrm{mmol} / \mathrm{L})$. Parathyroid hormone (PTH) was elevated at $12.6 \mathrm{pmol} / \mathrm{L}$ (reference range $1.6-6.9 \mathrm{pmol} / \mathrm{L}$ ), alkaline phosphatase was normal at $66 \mathrm{U} / \mathrm{L}$ (reference range $20-105 \mathrm{U} / \mathrm{L}), 24 \mathrm{~h}$ urinary calcium collection showed a calcium level of $9.8 \mathrm{mmol} / \mathrm{L}$ (reference range $2.5-7.5 \mathrm{mmol} /$ day), creatinine was $12.6 \mathrm{mmol} /$ day $(5.3-16.0 \mathrm{mmol} /$ day), the calcium/creatinine ratio was 0.78 (0.06-0.45). 25-Hydroxycholecalciferol was $67 \mathrm{nmol} / \mathrm{L}(50-150 \mathrm{nmol} / \mathrm{L})$. A parathyroid scan including pertechnate and Tc99m Sestamibi with early and delayed SPECT/CT of neck and mediastinum showed a solitary parathyroid adenoma in the lower pole of the left lobe of the thyroid (Fig. 1A). The patient underwent a minimally invasive parathyroidectomy, which revealed a very large superior parathyroid adenoma that was prolapsed into an inferior position. Histology confirmed a $3 \mathrm{~cm} \times 1.5 \mathrm{~cm} \times 1.2 \mathrm{~cm}$ gland-like structure weighing $2.5 \mathrm{~g}$ and appearing partially cystic (Fig. 1B). Microscopically a cellular parathyroid typical of an adenoma with no evidence of malignancy was found (Fig. 1C). Calcium and PTH levels normalised postoperatively.

Her past history includes anxiety and depression. She did not take any regular medications, and there is no reported family history of FIHP, HPT-JT syndrome, parathyroid adenomas or carcinomas. A computertomogram (CT) showed no ossifying fibromas of the jaw, and previous imaging showed no evidence of uterine or renal masses.

Three years post-operative, her son underwent genetic studies for developmental delay. A SNP chromosome microarray demonstrated a large deletion on chromosome 1 which led to further investigations in both parents. Our patient was subsequently found to have the same deletion as her son, encompassing the $C D C 73$ gene.
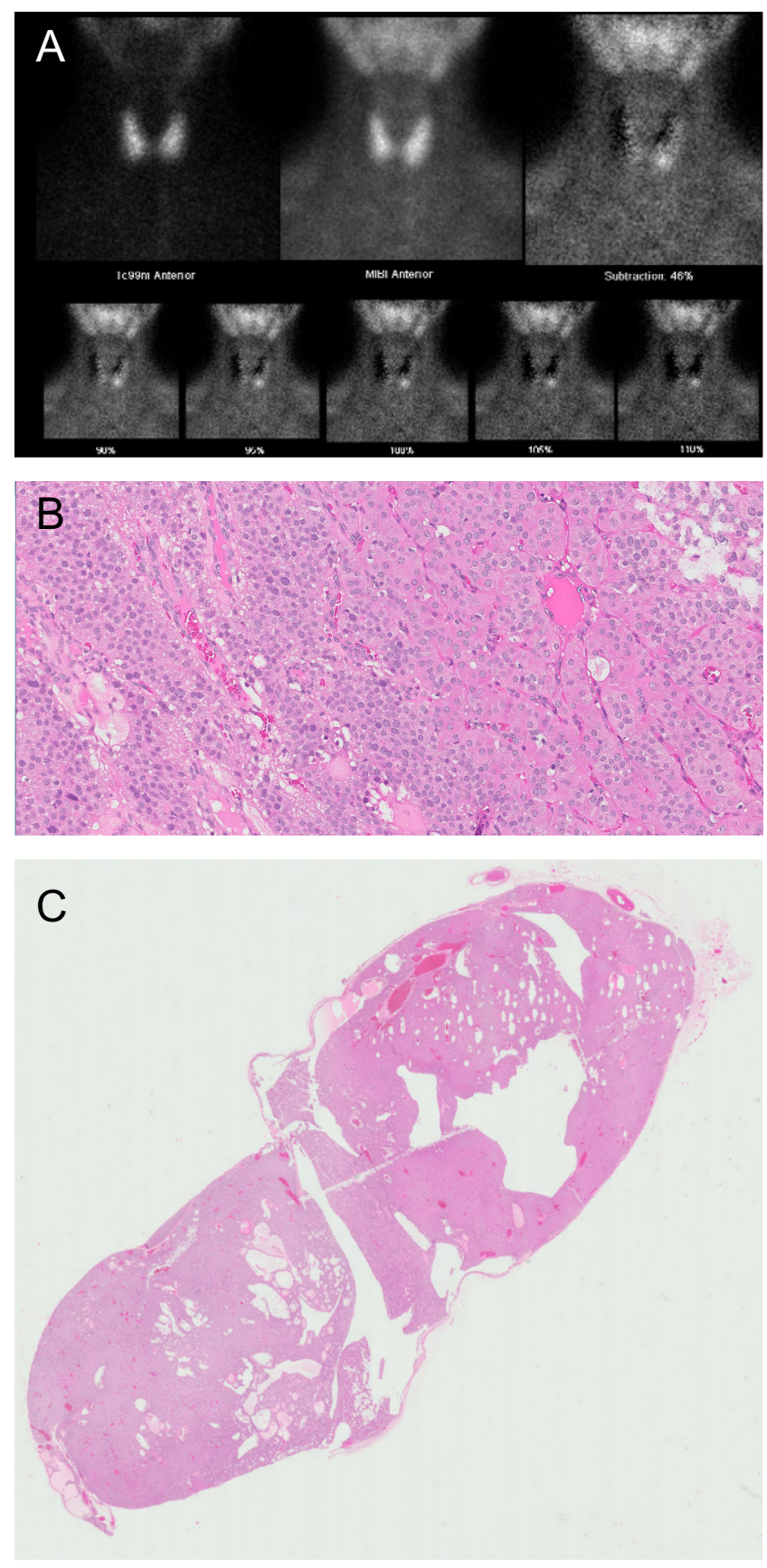

Figure 1

(A) Tc99m Sestamibi scan - subtraction images: solitary focus of intense Sestamibi accumulation at the lower pole of the left lobe of the thyroid; (B) left superior parathyroid measuring $3 \mathrm{~cm} \times 1.5 \mathrm{~cm} \times 1.2 \mathrm{~cm}$, (C) left superior parathyroid with sections showing a cellular parathyroid typical of an adenoma.

\section{Investigations}

DNA was extracted and purified from a peripheral blood sample. SNP microarray was performed using the Illumina Whole-Genome InfiniumCytoSNP 850K Array v1.1. All 
procedures for fragmentation, labelling and hybridisation were performed at the Australian Genome Research Facility (Melbourne, Australia) and were done according to the manufacturer's protocol (Illumina, San Diego, CA, USA). Raw data was analysed with BlueFuse Multi version 4.3 with an effective resolution of $200 \mathrm{~kb}$. The clinical significance of copy number variants (CNVs) greater than 200 kilobases was determined by comparison with public databases of copy number variants (i.e., DatabasE of Genomic variants and Phenotype in Humans Using Ensembl Resources (DECIPHER) and Database of Genomic Variants (DGV)). The UCSC genome browser February 2009 NCBI GRCh37/ hg19 assembly was used for comparison of genomic coordinates detected for each $\mathrm{CNV}$ called at the laboratory reporting threshold. The SNP microarray analysis in the patient (and son) identified an approximately $2.5 \mathrm{Mb}$ deletion at chromosome band 1q31.1-q31.2. The deleted region involved genomic coordinates chr1: 190750 491193265 225, and included 16 genes, of which only one is an OMIM listed disease-causing gene (CDC73).

\section{Treatment}

Treatment of our patient's hyperparathyroidism with a parathyroidectomy was performed years prior to her diagnosis of a novel, whole gene CDC73 deletion.

\section{Outcome and follow-up}

The patient is now undergoing regular surveillance for associated $C D C 73$ complications and has regular follow-up in the endocrinology clinic. This includes yearly regular PTH and calcium levels, yearly dental check-ups and periodic pelvis and renal tract ultrasounds.

\section{Discussion}

SNP microarray revealed a heterozygous, large deletion of the whole of the $C D C 73$ gene, which is the cause of $\mathrm{PH}$ in our patient. Literature review of databases including PubMed, Medline and Google Scholar regarding whole gene or intragenic CDC73 deletions was performed. In 2011, Cascón and coworkers reported the first case of a $C D C 73$ germline deletion in an 18-year-old woman presenting with hyperparathyroidism from a HPT-JT syndrome family demonstrating a whole gene deletion that involved 547040 bp with the location chr1: 192839 178-193 386218 on chromosome 1q31 (5). In 2012, a further report was published by Domingues and coworkers regarding a young man with primary hyperparathyroidism reported to have a
CDC73 whole gene deletion, as part of a larger chromosome deletion spanning $2.4-9.3 \mathrm{Mb}$ in size (6). In 2013, a larger French study was published by Bricaire and coworkers reporting molecular abnormalities in patients with PH. 20 index cases were identified and out of these 3 patients had a CDC73 gene deletion with only one of them expanding beyond the gene. All three were women and all presented with parathyroid carcinoma and had at least one feature of HPT-JT (7). Mehta and coworkers described seven members from a single HPT-JT family with a whole gene deletion of CDC73; however, the extent of the deletion was not reported (8). In 2017, Rubinstein and coworkers published a case report of a woman with HPT-JT syndrome who was found to have a $4.1 \mathrm{Mb}$ deletion on chromosome 1q31.2-q31.3 (chr1: 192127 840-196 227 528), including the CDC73 gene (9). The large gene deletion in our patient is similar to the one reported by Rubinstein and coworkers that had already highlighted the possibility that deleted genes (other than the CDC73) may be associated with developmental delay syndromes. The deletions in both cases partially overlap with known 1q deletions, encompassing the 1q25-32 region including the $C D C 73$ gene. Interestingly, the most common clinical manifestation of these $1 \mathrm{q}$ deletions is developmental delay (9). This is especially noteworthy as developmental delay in our patient's son had led to genetic investigations in the first place. Recently Mamedova and coworkers enrolled 65 patients with $\mathrm{PH}$ and screened for possible underlying genetic mutations. One female patient with parathyroid carcinoma had a whole gene deletion of $C D C 73$, and one male patient had a deletion of exons $1-10$ of the $C D C 73$ gene with an atypical parathyroid adenoma (10).

Interestingly, and in contrast to the aforementioned female patient reports, our patient did not have a parathyroid carcinoma and does not have a known family history of HPT-JT syndrome or other clinical features of HPT-JT at this point in time. Furthermore, the incidental diagnosis of a $C D C 73$ gene deletion, which was detected during investigations for developmental delay in her son is novel. This may suggest that young patients presenting with hypercalcaemia even without features of HPT-JT syndrome, or a FIHP phenotype, should be considered for genetic screening including CDC73 mutations and deletions. The diagnosis of an underlying $C D C 73$ deletion was delayed in this case and was an incidental finding. However, early detection of possible underlying genetic aetiology has a significant impact on surveillance programmes and also genetic counselling for the patient and their families, and it is important that clinicians have a low threshold for genetic screening in young patients presenting with hyperparathyroidism. 
Clinicians should use established guidelines for the diagnosis, genetic screening and treatment of hyperparathyroidism. Baseline serum PTH, 25-hydroxycholecalciferol, calcium, creatinine and a 24-h urinary calcium should be assessed. Furthermore, bone mineral density assessment and abdominal imaging for nephrocalcinosis should be performed. Once the diagnosis of PH is established, localisation studies should be performed and will depend on local expertise but can include cervical ultrasonography, ${ }^{99 m}$ Technetium Sestamibi scintigraphy or 4-dimensional CT. A thorough family history needs to be taken and patients should be assessed for a possible underlying genetic syndrome. Parathyroidectomy should be considered for all symptomatic patients, serum calcium levels greater than $1 \mathrm{mg} / \mathrm{dL}(0.25 \mathrm{mmol} / \mathrm{L})$ above the upper limit of the reference interval for total calcium, when there is evidence of renal involvement including nephrocalcinosis, hypercalciuria $(24 \mathrm{~h}$ urinary calcium level $>400 \mathrm{mg} / \mathrm{dL}$ ) or glomerular filtration rate $<60 \mathrm{~mL} / \mathrm{min}$. Patients with osteoporosis or fragility fractures and all patients aged $<50$ years regardless of objective or subjective features are also candidates for parathyroidectomy $(11,12)$.

\section{Declaration of interest}

The authors declare that there is no conflict of interest that could be perceived as prejudicing the impartiality of the research reported.

\section{Funding}

This research did not receive any specific grant from any funding agency in the public, commercial or not-for-profit sector

\section{Patient consent}

Written consent has been obtained from our patient for this article and all accompanying images.

\section{Author contribution statement}

Caroline Bachmeier has reviewed our patient in clinic as her role as an endocrinology advanced trainee and is the main author of the manuscript. Dr Kanowski has reported the initial parathyroidectomy and has supplied the histology photographs of this case report and gave advice on the histopathology reporting. Dr Patel reviewed our patient in his role as a consultant geneticist. He was also responsible for the genetic testing and has greatly helped in writing the genetics part of this case report. A/Prof. Dr Sangla is the overseeing consultant at Townsville Hospital and has coordinated and overseen the care of our patient. He has helped with writing this manuscript.

\section{References}

1 Fraser WD. Hyperparathyroidism. Lancet 2009374 145-158. (https:// doi.org/10.1016/S0140-6736(09)60507-9)

2 Marx SJ. Hyperparathyroid and hypoparathyroid disorders. New England Journal of Medicine 2000343 1863-1875. (https://doi. org/10.1056/NEJM200012213432508)

3 Newey PJ, Bowl MR, Cranston T \& Thakker RV. Cell division cycle protein 73 homolog (CDC73) mutations in the hyperparathyroidism-jaw tumor syndrome (HPT-JT) and parathyroid tumors. Human Mutation 201031 295-307. (https://doi.org/10.1002/ humu.21188)

4 Carpten JD, Robbins CM, Villablanca A, Forsberg L, Presciuttini S, Bailey-Wilson J, Simonds WF, Gillanders EM, Kennedy AM, Chen JD, et al. HRPT2, encoding parafibromin, is mutated in hyperparathyroidism-jaw tumor syndrome. Nature Genetics 200232 676-680. (https://doi.org/10.1038/ng1048)

5 Cascón A, Huarte-Mendicoa CV, Javier Leandro-García L, Letón R, Suela J, Santana A, Costa MB, Comino-Méndez I, Landa I, Sánchez L, et al. Detection of the first gross CDC73 germline deletion in an HPT-JT syndrome family. Genes, Chromosomes and Cancer 201150 922-929. (https://doi.org/10.1002/gcc.20911)

6 Domingues R, Tomaz RA, Martins C, Nunes C, Bugalho MJ \& Cavaco BM. Identification of the first germline HRPT2 whole-gene deletion in a patient with primary hyperparathyroidism. Clinical Endocrinology 201276 33-38. (https://doi.org/10.1111/j.13652265.2011.04184.x)

7 Bricaire L, Odou MF, Cardot-Bauters C, Delemer B, North MO, Salenave S, Vezzosi D, Kuhn JM, Murat A, Caron P, et al. Frequent large germline HRPT2 deletions in a French National cohort of patients with primary hyperparathyroidism. Journal of Clinical Endocrinology and Metabolism 201398 E403-E408. (https://doi. org/10.1210/jc.2012-2789)

8 Mehta A, Patel D, Rosenberg A, Boufraqech M, Ellis RJ, Nilubol N, Quezado MM, Marx SJ, Simonds WF \& Kebebew E. Hyperparathyroidism-jaw tumor syndrome: results of operative management. Surgery 2014156 1315-1324; discussion 1324-1315. (https://doi.org/10.1016/j.surg.2014.08.004)

9 Rubinstein JC, Majumdar SK, Laskin W, Lazaga F, Prasad ML, Carling T \& Khan SA. Hyperparathyroidism-jaw tumor syndrome associated with large-scale 1q31 deletion. Journal of the Endocrine Society 20171 926-930. (https://doi.org/10.1210/js.2016-1089)

10 Mamedova E, Mokrysheva N, Vasilyev EV, Petrov V, Pigarova E, Kuznetsov S, Kuznetsov N, Rozhinskaya L, Melnichenko GA Dedov I, et al. Primary hyperparathyroidism in young patients in Russia: high frequency of hyperparathyroidism-jaw tumour syndrome. Endocrine Connections 20176 557-565. (https://doi org/10.1530/EC-17-0126)

11 Khan AA, Hanley DA, Rizzoli R, Bollerslev J, Young JE, Rejnmark L, Thakker R, D'Amour P, Paul T, Van Uum S, et al. Primary hyperparathyroidism: review and recommendations on evaluation, diagnosis, and management. A Canadian and international consensus. Osteoporosis International 201728 1-19. (https://doi. org/10.1007/s00198-016-3716-2)

12 Wilhelm SM, Wang TS, Ruan DT, Lee JA, Asa SL, Duh QY, Doherty GM, Herrera MF, Pasieka JL, Perrier ND, et al. The American Association of Endocrine Surgeons Guidelines for definitive management of primary hyperparathyroidism. JAMA Surgery 2016 151 959-968. (https://doi.org/10.1001/jamasurg.2016.2310)
Received in final form 9 February 2018 Accepted 19 February 2018 